\title{
Moyamoya Disease In The Differential Diagnosis Of Multiple Sclerosis: A Case Report
}

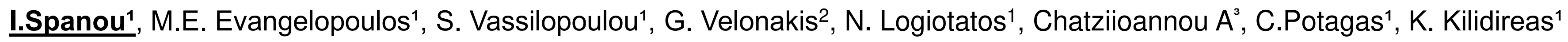

${ }^{1}$ Department of Neurology, Eginition Hospital, National and Kapodistrian University of Athens, Greece, ${ }^{2}$ Research Unit of Radiology and Medical Imaging, University of Athens Medical School, Athens, Greece, ${ }^{3}$ Department of Radiology, Aretaion Hospital, National and Kapodistrian University of Athens, Greece

\section{Introduction}

Moyamoya disease (MMD) manifestation may be insidious in young female patients presenting with unspecific neurological symptoms and atypical white matter lesions in MRI, mimicking Multiple Sclerosis.

\section{The Patient}

- 44-year old woman

- For years, complaining of intermittent weakness of her left arm- attributed to psychosomatic problems.

\section{History \& Examination}

- Transient numbness of her right-sided face \& arm with small brain lesions in MRI (Fig.A)

- Unremarkable except from plantar and jerky deep tendon reflexes.

\section{Investigation}

- MRA \& DSA: revealed findings typically seen in MMD (Fig. B, C, D).

\section{Management}

- Antiplatelet therapy was initiated.

- Few days later, developed suddenly global aphasia \& right hemiparesis (NIHSS 6).

- MRI revealed an acute infarct in the distribution of the left MCA (Fig. E, F).

- Thrombolysis was not performed due to the increased risk of hemorrhage in MMD patients.

At discharge: NIHSS 3.
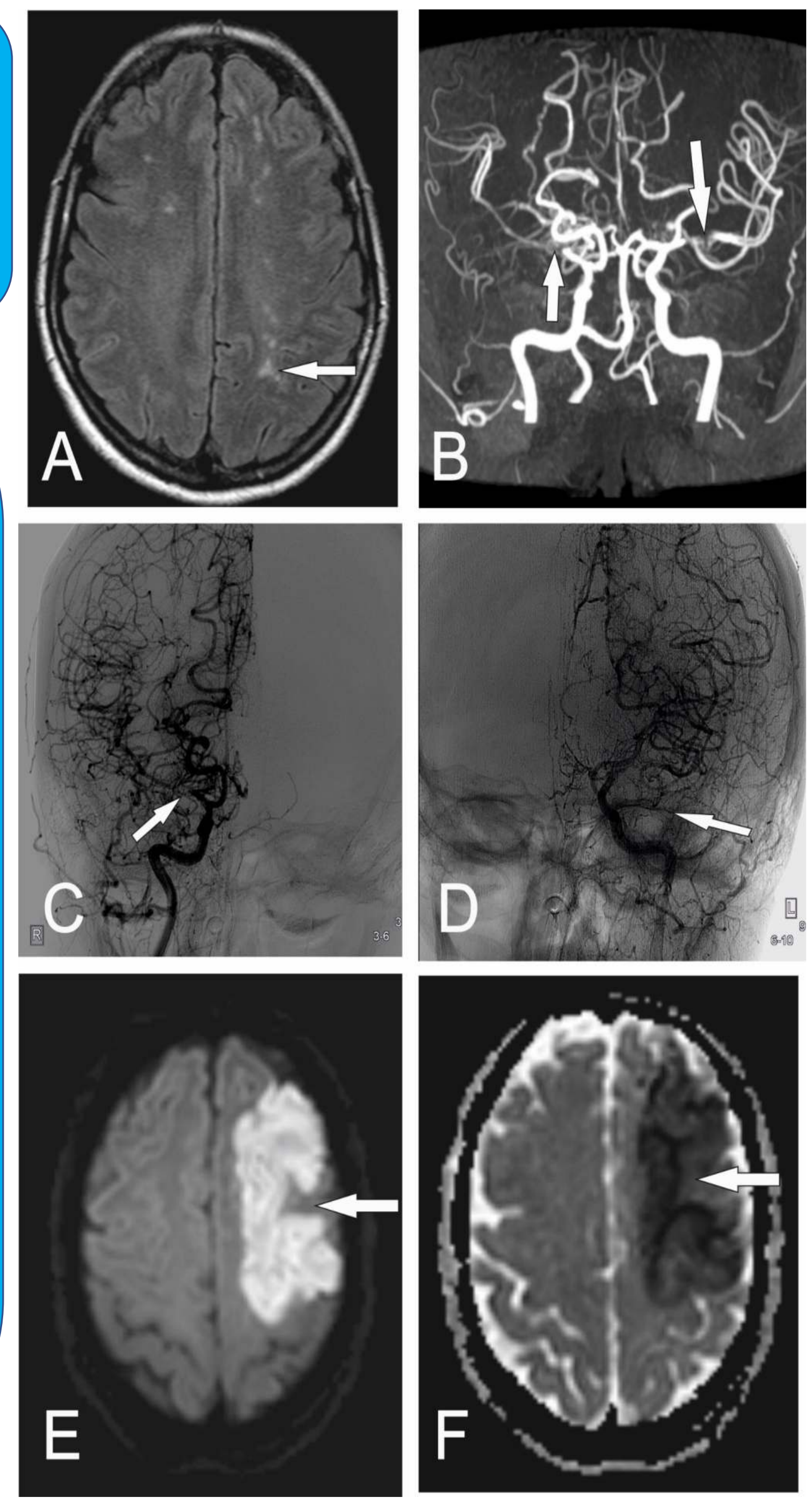

\section{Discussion}

- In young patients with transient neurological symptoms suggestive of Multiple Sclerosis and atypical, multifocal white matter lesions in MRI, MMD should be considered in the differential diagnosis.

- The key to diagnosis of MMD is imaging of intracranial vasculature. 